\title{
Análisis Comparativo Estructural Mediante Galgas Uniaxiales y Triaxiales para un Perfil de Ala NACA 4412
}

\author{
Bernabé Ramírez ${ }^{*}$ Ana M. Castañeda y Víctor Nuño \\ Universidad Autónoma de Baja California, Facultad De Ingeniería, Blvd. Benito Juárez s/n Col: Ex Ejido \\ Coahuila, Mexicali Baja California, México (e-mail: bernabe.ramirez@uabc.edu.mx, \\ ana.castaneda@uabc.edu.mx,vnuno@uabc.edu.mx)
}

* Autor a quien debe ser dirigida la correspondencia

Recibido Dic. 13, 2013; Aceptado Feb. 20, 2014; Versión final recibida Abr. 17, 2014

\begin{abstract}
Resumen
Se realizó un estudio sobre un ala con perfil NACA 4412 construido en aluminio 6061, para obtener mediciones de esfuerzos del perfil aerodinámico, que se procesaron por medio de un sistema de adquisición de datos PXI. Se realizó un análisis comparativo entre lo teórico, el análisis por el método de elementos finitos usando el programa ANSYS y medido con el sistema de adquisición de datos PXI, para validar la metodología utilizada en la medición. Mediante galgas uniaxiales se logró la configuración de galgas triaxiales, integrando este arreglo al sistema de adquisición de datos, para monitorear la información medida. Según los resultados teóricos, los de ANSYS y los experimentales, se observa un aceptable grado de exactitud entre los valores. Estos resultados validan la adquisición de datos por medio de una configuración de galgas en forma de roseta en delta para el análisis de esfuerzos del perfil aerodinámico.
\end{abstract}

Palabras claves: método del elemento finito, galgas extensométricas, esfuerzos, perfil aerodinámico, deformación unitaria.

\section{Comparative Structural Analysis by Uniaxial and Triaxial Strain Gauges on a Wing Profile NACA 4412}

\begin{abstract}
A study was performed on the structural behavior of a wing profile NACA 4412 fabricated with aluminum 6061, to obtain stress measurements of an airfoil, which are processed by a data acquisition system PXI. A comparative analysis between theoretical estimates, analysis by the finite elements method using the software ANSYS and values measured with the data acquisition system PXI, for validating the methodology used in the measurement. Through a set of uniaxial gauges, a triaxial configuration was achieved, integrating this arrangement to the data acquisition system in order to monitor the information measured. According to the theoretical results, those of the ANSYS analysis and the experimental values, an acceptable degree of accuracy was achieved. These results validate the data acquisition through a setting gauge in the form of delta rosette for the stress analysis of an airfoil.
\end{abstract}

Keywords: finite element method, strain gauges, stress, airfoil, strain. 


\section{INTRODUCCIÓN}

Conforme se incrementa la demanda en la industria aeroespacial, mayores son los requerimientos de análisis sobre los componentes de las aeronaves, generando la necesidad de estudios que puedan agilizar el proceso de su evaluación estructural. El siguiente estudio consiste en un análisis preliminar, realizado para determinar las similitudes y discrepancias entre los resultados del análisis teórico y el experimental, con lo cual se pueda generar una metodología de simplificación en el estudio estructural del ala de una aeronave. Hoy en día uno de los temas que más atraen la atención del trabajo de investigación sobre el análisis y diseño en ingeniería, es precisamente su aspecto metodológico. En décadas pasadas, los libros sobre diseño mecánico hacían referencia en su parte introductoria a alguna metodología de diseño describiéndola de manera muy generalizada. Comúnmente la enseñanza formalizada de diseño mecánico se ajustaba solo al cálculo de elementos de máquinas, y en el mejor de los casos, los ingenieros de diseño, de acuerdo a su experiencia personal adquirida durante el ejercicio de la profesión, desarrollaban su propia metodología.

Actualmente, debido a la importancia que se ha descubierto en torno a la metodología de diseño, se investiga sobre las mejores formas de proceder y se publican libros enteros sobre este tema (Watanave y Urriolagoitia, 1986) teniendo como base sustentable para estos estudios, que el diseño mecánico hace uso de las mecánicas aplicadas a la ingeniería, las ciencias de los materiales y las matemáticas (Shigley y Mitchell, 1986). En el caso del análisis estructural una de las herramientas de gran ayuda ha sido el método del elemento finito que consiste en dividir la geometría en la que se quiere resolver una ecuación diferencial de un campo escalar o vectorial en un dominio, en pequeños elementos, teniendo en cuenta las ecuaciones de campo en cada elemento, los elementos del entorno de vecindad y las fuentes generadoras de campo (Escribano 2007).

Aunado a esto el desarrollo de sistemas computacionales ha propiciado llevar el campo de la instrumentación electrónica a la instrumentación virtual cuyo fin es introducir metodologías de aprendizaje de varios campos de la ingeniería utilizando las nuevas herramientas de programación gráfica. Las variables o magnitudes físicas susceptibles de ser transformadas en señales eléctricas son muy numerosas y eso da lugar, a que también sean muy variadas las tecnologías o principios de transducción (inductivo, piezoeléctrico, extensiométrico, capacitivo, etc.), que se emplean actualmente (Mandado 1996). La instrumentación virtual es un entorno de programación gráfico que nos permite el control y la simulación de cualquier instrumento local o remoto y, a su vez, una herramienta potente de adquisición y procesado de señales, su utilización no queda exclusivamente limitada a las disciplinas electrónicas, sino que puede hacerse extensiva a otras especialidades (Manuel 2002).

Debido a que la industria de la aviación demanda combinaciones de una elevada resistencia, bajo peso y grandes relaciones de rigidez/peso, se ha estimulado la investigación en todos los países industriales y las últimas aleaciones basadas en el sistema aluminio-litio están en su etapa inicial de aplicación. Ofrecen los beneficios de que no solo satisfacen los requerimientos de resistencia, sino que además tiene un módulo de elasticidad mayor, lo que permite producir elementos estructurales más rígidos con significativos ahorros en el peso (King, 1992). A medida que la tecnología de materiales avanza, también lo han hecho las distintas aleaciones, como las aleaciones ligeras de aluminio y otras aleaciones basadas en este, que hasta el día de hoy han ido aumentando su resistencia mecánica y disminuyendo su peso, normalmente vienen en forma de planchas de diferentes espesores, que se las trabaja cortando, doblando y perforando para darles las formas necesarias, en determinados casos incluso de bloques enteros se llega a mecanizar piezas constructivas y estructurales. (Subauste, 2009).

En otros trabajos desarrollados también se utiliza el MEF y galgas extensométricas como medio de validación para los resultados obtenidos (Gualda et al., 2010), así como análisis comparativos entre ambos métodos (Akça et al., 2002). Otro autor (Reymer, 2011) también realiza un análisis de elemento finito validado a través de galgas extensométricas sobre un componente aerodinámico. Tomando lo anterior en consideración, a través de este estudio se busca una metodología que ayude en el análisis de formas complejas tales como la estructura de un ala de avión (en este caso se trabajo con un perfil aerodinámico NACA 4412 fabricado en aluminio 6061), tratando de simplificar su estudio obteniendo resultados que se puedan someter a discusión.

\section{DISEÑO EXPERIMENTAL}

En la parte de la experimentación primero se realizó un estudio comparativo entre el análisis teórico, el análisis de elemento finito por medio del programa ANSYS y el sistema de adquisición de datos PXI con galgas individuales, para determinar los porcentajes de error que existen entre estos métodos se tomaron en cuenta las deformaciones producidas en un ensayo de flexión sobre un elemento estructural de aluminio 
6061 de superficie recta, con una sección transversal rectangular de $19.05 \mathrm{~mm}$ de base y $12.7 \mathrm{~mm}$ de altura y un largo de $600 \mathrm{~mm}$. Para determinar las deformaciones teóricas sufridas en la barra sujeta a flexión se tomó en consideración el desplazamiento (W) que sufre la pieza de prueba al actuar un par de cargas iguales sobre ella a una distancia de $150 \mathrm{~mm}$ respecto a la sección central, midiendo este desplazamiento por medio de un micrómetro de carátula, obteniendo los resultados a partir de la curvatura presentada, la figura 1 muestra las variables utilizadas para la determinación de estos cálculos.

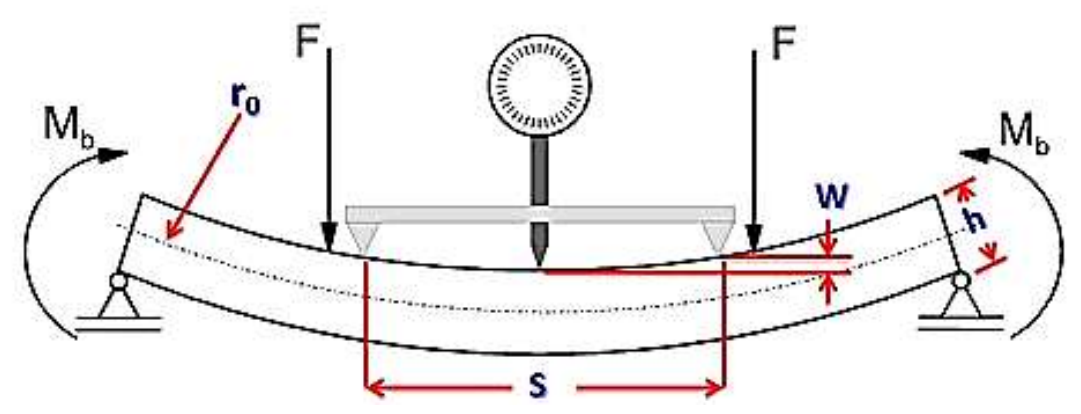

Fig. 1 Consideraciones para el cálculo de deformaciones en el ensayo de flexión.

Para la obtención de las deformaciones teóricas mediante el ensayo de flexión se consideraron los siguientes modelos:

$\varepsilon=\frac{\sigma}{E}=\frac{h}{2 r_{0}}$

$r_{0}=\frac{W}{2}+\frac{s^{2}}{8 W}$

Siendo ' $\varepsilon$ ' la deformación unitaria, ' $\sigma$ ' el esfuerzo, 'E' el módulo de elasticidad, ' $h$ ' la altura de la barra, ' $r o$ ' radio de la barra deformada, 'W' el desplazamiento y 'S' la sección de la curva del desplazamiento.

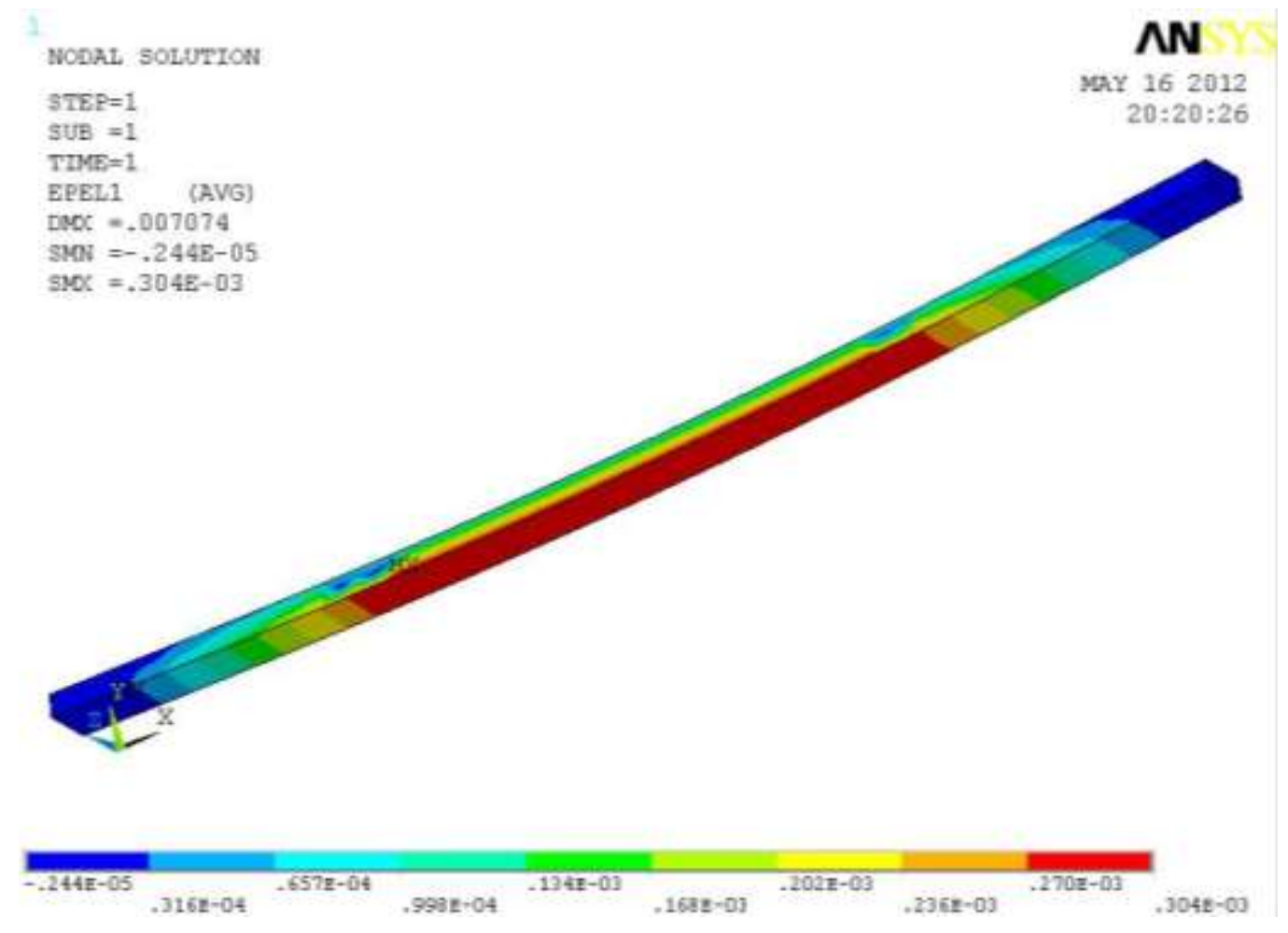

Fig. 2 Deformación obtenida a través de ANSYS para una $\mathrm{w}=0.3 \mathrm{~mm}$ 
Tabla 1. Comparación de los porcentajes de error obtenidos en las deformaciones del perfil rectangular por los tres diferentes experimentos realizados

\begin{tabular}{|c|c|c|c|c|c|}
\hline $\mathrm{W}(\mathrm{mm})$ & $\begin{array}{l}\text { Deformación } \\
\text { Teórica }(\mu \varepsilon)\end{array}$ & $\begin{array}{l}\text { Deformación } \\
\text { ANSYS }(\mu \varepsilon)\end{array}$ & $\begin{array}{l}\text { Deformación } \\
\operatorname{PXI}(\mu \varepsilon)\end{array}$ & $\begin{array}{c}\% \text { Error } \\
\text { Teórica/ANSYS }\end{array}$ & $\begin{array}{c}\text { \% Error } \\
\text { Teórica/PXI }\end{array}$ \\
\hline 0.3 & 298 & 301 & 270 & $1 \%$ & $9 \%$ \\
\hline 0.35 & 347 & 350 & 370 & $1 \%$ & $-7 \%$ \\
\hline 0.4 & 397 & 400 & 413 & $1 \%$ & $-4 \%$ \\
\hline 0.45 & 446 & 470 & 462 & $5 \%$ & $-4 \%$ \\
\hline 0.5 & 496 & 501 & 479 & $1 \%$ & $3 \%$ \\
\hline 0.55 & 545 & 555 & 517 & $2 \%$ & $5 \%$ \\
\hline 0.6 & 595 & 601 & 586 & $1 \%$ & $2 \%$ \\
\hline 0.65 & 645 & 651 & 664 & $1 \%$ & $-3 \%$ \\
\hline 0.7 & 694 & 701 & 722 & $1 \%$ & $-4 \%$ \\
\hline 0.75 & 744 & 751 & 743 & $1 \%$ & $0 \%$ \\
\hline 0.8 & 793 & 801 & 782 & $1 \%$ & $1 \%$ \\
\hline
\end{tabular}

Para los resultados obtenidos experimentalmente se utilizó una galga extensométrica formando $1 / 4$ de puente de Wheatstone, en posición paralela a las deformaciones, con una resistencia de $120 \Omega$ y con un factor de galga 2.07 para propósitos generales, el equipo utilizado para la instalación consistió en desengrasante, acondicionador, neutralizador, acelerador de pegado y pegamento loctite, así como estaño y cautín para la conexión de la galga, interconectándose al módulo para adquisición de datos de National Instruments NI 9237 utilizado para medir deformaciones, el programa utilizado para el procesamiento de las señales fue LabView en el cual se obtuvo el promedio de un conjunto de muestras tomadas en un determinado tiempo.

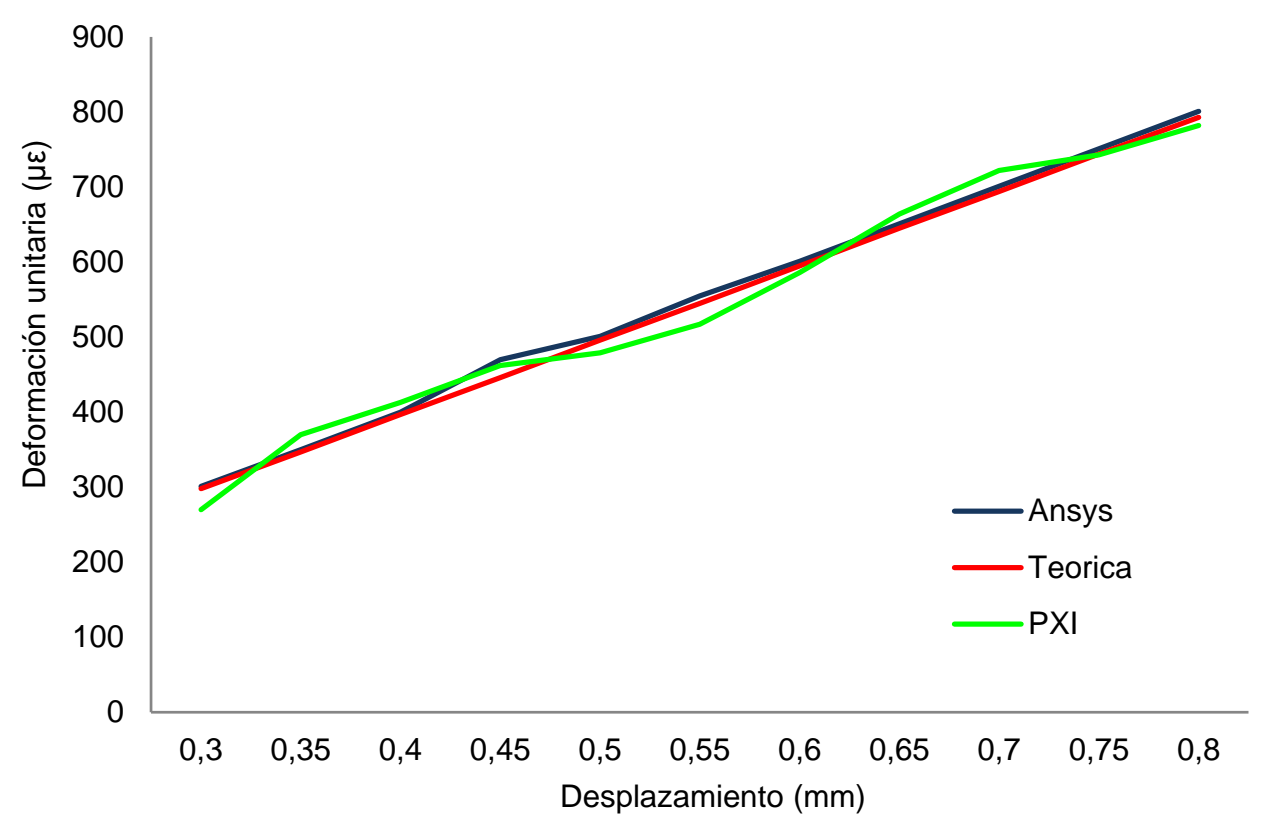

Fig. 3: Valores teóricos, simulados y experimentales

Para la obtención de resultados simulados se utilizó el programa ANSYS clásico versión 2011, llevando a cabo el método del elemento finito modelando la barra en tres dimensiones, se realizó el análisis con un mallado medio y uno fino sobre la variable de tensión con una diferencia menor al $0.5 \%$, que de acuerdo a (Chagoyén et al., 2013), esta es una buena precisión. Se definieron las condiciones de contorno de acuerdo a la figura 1 colocando el empotramiento en los extremos de la barra y aplicando diferentes fuerzas, donde para un desplazamiento de $0.3 \mathrm{~mm}$ el par de fuerzas correspondiente fue $244.36 \mathrm{~N}$ cada una, incrementando el desplazamiento sufrido en $0.05 \mathrm{~mm}$ hasta llegar a los $0.8 \mathrm{~mm}$ con un par de fuerzas de $650.26 \mathrm{~N}$ cada una, considerando el material elástico lineal, isotrópico y homogéneo, utilizando un tipo de elemento sólido, tetraédrico de 10 nodos, en la figura 2 se puede observar la deformación para un desplazamiento $\mathrm{w}=0.3 \mathrm{~mm}$. Debido a las condiciones bajo las cuales se realizó la experimentación y respaldado por los resultados teóricos obtenidos, no se considera la existencia de fuentes de no linealidades. 
En la tabla 1 se muestran los resultados de las deformaciones obtenidas de la primera etapa de experimentación con el perfil estructural rectangular, permitiendo hacer una comparación entre el análisis teórico, el análisis del programa ANSYS y el sistema de adquisición de datos PXI con galgas individuales, donde se puede apreciar el porcentaje de error presentado en los experimentos realizados. En la figura 3 se muestra de manera gráfica la comparación de los tres resultados, donde el eje vertical representa las deformaciones en microstrains y el eje horizontal los desplazamientos en milímetros.

Una vez determinados los porcentajes de error para galgas individuales sobre un elemento estructural de superficie recta con sistema de cargas axiales se consideró conveniente evaluar un análisis de galgas extensométricas triaxiales ya que a través de estas se pueden obtener mediciones de superficies no rectas como un perfil aerodinámico en donde el sistema de cargas no es unidireccional. Primeramente se procedió con la fabricación de un perfil aerodinámico NACA 4412, en material de aluminio 6061, la configuración del ala fue recta con una longitud de $304.8 \mathrm{~mm}$, en vista transversal el perfil tiene una altura de $9.5 \mathrm{~mm}$ y un ancho de $76 \mathrm{~mm}$. Utilizando el mismo material, equipo y procedimiento de pegado de la experimentación anterior, se colocaron 3 galgas uniaxiales en configuración delta sobre el perfil aerodinámico para obtener las deformaciones sufridas en el material a una distancia de $25.4 \mathrm{~mm}$ con respecto al empotramiento. Las galgas fueron colocadas sobre el perfil en un ángulo de $120^{\circ}$ una respecto a la otra, para posteriormente realizar la conexión de cada una de ellas como se aprecia en la figura 4, y llevar a cabo el análisis de las mediciones con el sistema de adquisición de datos.

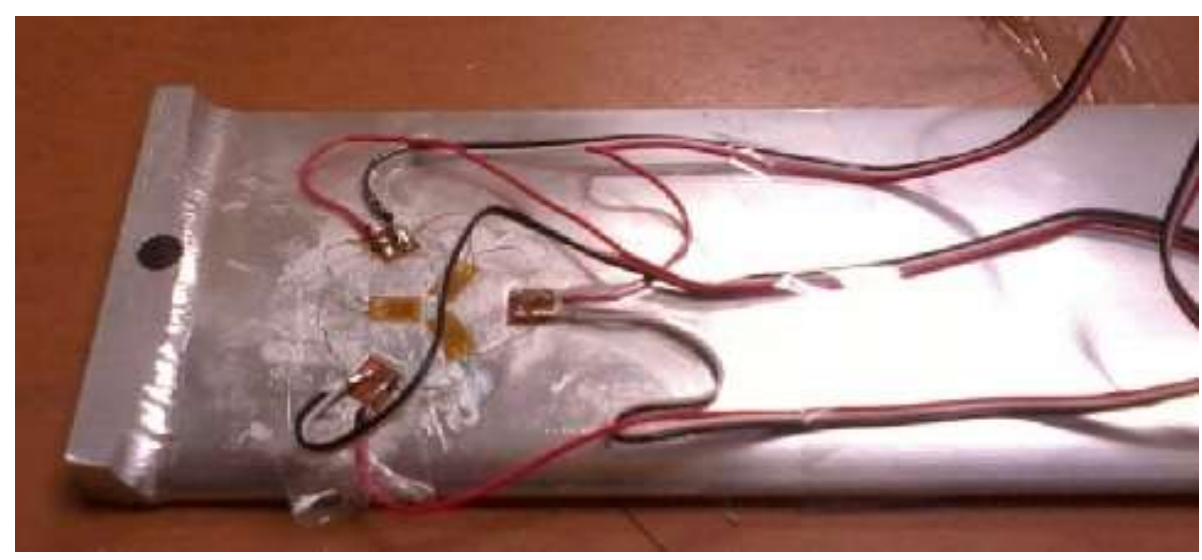

Fig. 4 Conexión de galgas en configuración roseta delta

Terminadas las conexiones de las galgas se llevó a cabo el análisis real del perfil NACA 4412, realizando la experimentación en condiciones de viga en voladizo como se aprecia en la figura 5 , empotrada en un extremo y aplicando fuerzas de flexión en el otro de $11.33 \mathrm{~N}, 21.14 \mathrm{~N}, 30.95 \mathrm{~N}$ y $40.76 \mathrm{~N}$ por medio de cuatro cargas diferentes y debido a estas se obtuvo una serie de lecturas de las deformaciones producidas que ayudaron a determinar el esfuerzo sufrido con cada una de ellas.

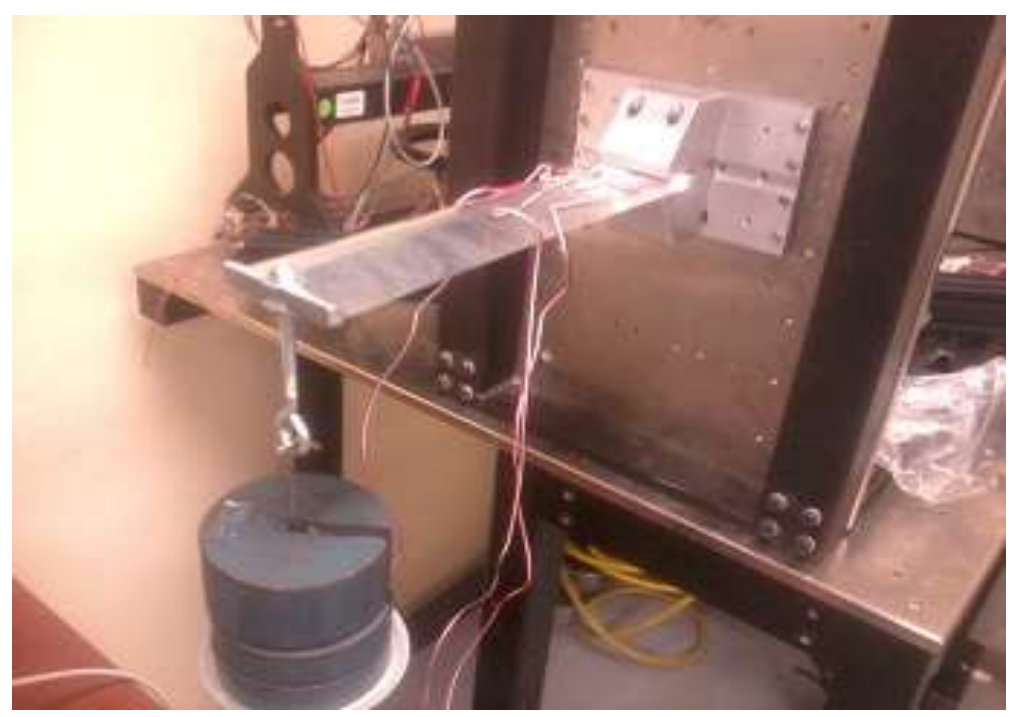

Fig. 5 Perfil NACA 4412 en voladizo, sujeto a diferentes cargas. 
Por último se realizó el análisis por medio del elemento finito para hacer la comparación con los datos obtenidos por el sistema de adquisición de datos y las galgas triaxiales. En este caso debido a la complejidad de la geometría se realizó el análisis en ANSYS Workbench procurando realizarlo bajo los mismos parámetros que en el experimento anterior, comprobando buena precisión en los resultados y descartando fuentes de no linealidades. En la figura 6 se muestra el campo de esfuerzos obtenido a través de ANSYS. Para el análisis experimental se tomó en cuenta la configuración de las galgas, debido a que es necesario utilizar las ecuaciones siguientes que involucran todos los desplazamientos para encontrar el esfuerzo máximo.

Maximum Principal Stress

Type: Maximum Principal Stress

Unit: MPa

Time: 1

08/04/2013 06:33 p.m.

\begin{tabular}{|l|}
$\mathbf{1 5 . 9 5 1}$ Max \\
13.706 \\
11.462 \\
9.2168 \\
6.9719 \\
4.727 \\
2.4821 \\
0.23726 \\
-2.0076 \\
$\mathbf{- 4 . 2 5 2 5}$ Min
\end{tabular}
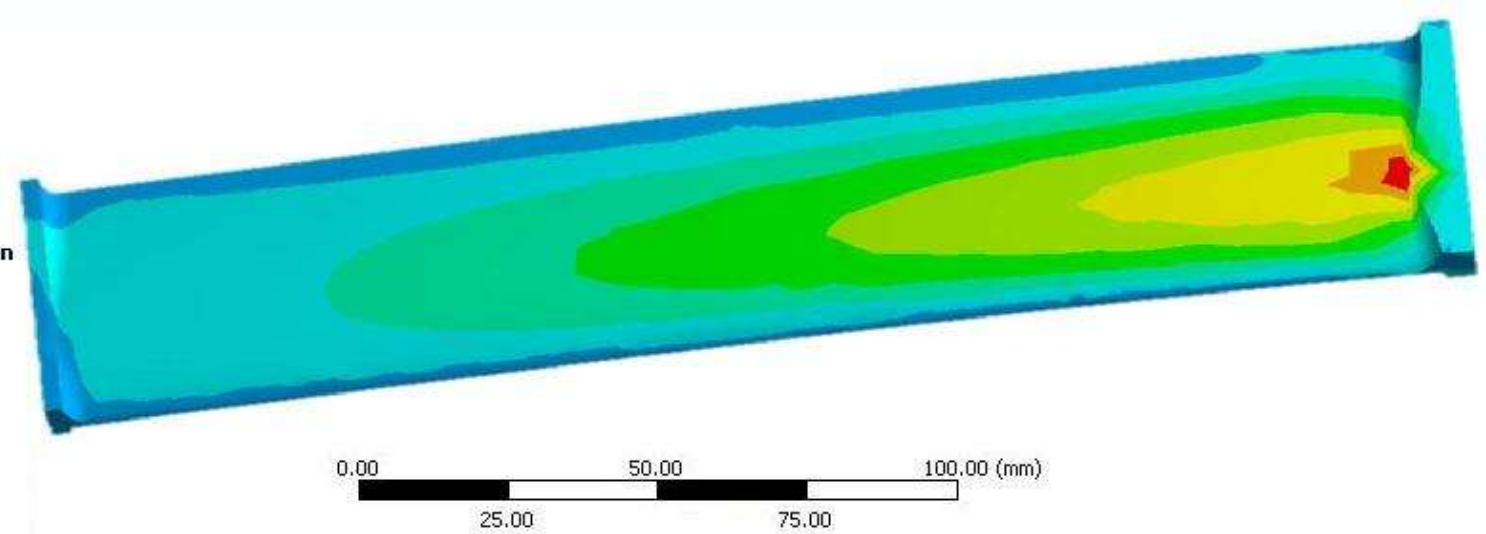

Fig. 6 Campo de esfuerzos obtenido a través de ANSYS.

$\varepsilon_{\max -\min }=\frac{\varepsilon_{1}+\varepsilon_{2}+\varepsilon_{3}}{3} \pm \frac{\sqrt{2}}{3} \sqrt{\left[\left(\varepsilon_{1}-\varepsilon_{2}\right)^{2}+\left(\varepsilon_{2}-\varepsilon_{3}\right)^{2}+\left(\varepsilon_{3}-\varepsilon_{1}\right)^{2}\right]}$

$T_{\max }=\frac{E}{1-\mu^{2}}\left(\varepsilon_{\max }+\mu \varepsilon_{\min }\right)$

Done 'Tmax' es el esfuerzo resultante máximo y ' $\mu$ ' es la relación de Poisson, los demás símbolos fueros descritos en la ecuación 1.

A partir de estas ecuaciones y con la aplicación de las diferentes cargas sobre nuestro perfil aeroespacial se obtuvieron los resultados experimentales.

Autores como (Kacmarcik et al., 2010), así como (Gröning et al., 2012) realizaron la validación de sus análisis numéricos mediante la utilización de galgas extensométricas triaxiales.

\section{RESULTADOS}

Los resultados experimentales que se obtuvieron por medio del sistema de adquisición de datos y la configuración de galgas a partir del análisis en voladizo del perfil NACA 4412 se observan en la tabla 2.

Tabla 2. Resultados medidos por el sistema de adquisición de datos a través de galgas en configuración triaxial

\begin{tabular}{|l|c|c|c|c|}
\hline & $\varepsilon_{1}$ & $\varepsilon_{2}$ & $\varepsilon_{3}$ & $\begin{array}{c}\text { Esfuerzo Máximo } \\
(\mathrm{MPa})\end{array}$ \\
\hline Carga 1 & $5.78 \mathrm{E}-06$ & $1.98 \mathrm{E}-05$ & $8.96 \mathrm{E}-06$ & 4.71 \\
\hline Carga 2 & $1.13 \mathrm{E}-05$ & $4.83 \mathrm{E}-05$ & $1.92 \mathrm{E}-05$ & 11.24 \\
\hline Carga 3 & $2.89 \mathrm{E}-05$ & $7.72 \mathrm{E}-05$ & $3.35 \mathrm{E}-05$ & 18.48 \\
\hline Carga 4 & $5.76 \mathrm{E}-05$ & $9.86 \mathrm{E}-05$ & $6.45 \mathrm{E}-05$ & 25.54 \\
\hline
\end{tabular}


Una vez obtenidos los resultados experimentales de las mediciones reales se procedió a realizar el análisis del elemento finito a través de ANSYS para la determinación del esfuerzo sufrido en el ala debido a la aplicación de las 4 diferentes cargas mencionadas anteriormente. Obteniendo el valor del esfuerzo en la zona donde se pegaron las galgas. Los resultados obtenidos por medio de ANSYS para la aplicación de las cuatro diferentes cargas se muestran en la tabla 3, y la comparación del análisis experimental por medio de las galgas triaxiales y el sistema PXI con respecto al análisis numérico experimental por medio de ANSYS se observa en la tabla 4.

Tabla 3. Resultados teóricos obtenidos del ala en ANSYS

\begin{tabular}{|c|c|c|c|}
\hline & Peso & Fuerza & Esfuerzo \\
\hline Carga 1 & $1.154 \mathrm{Kg}$ & $11.33 \mathrm{~N}$ & $4.22 \mathrm{MPa}$ \\
\hline Carga 2 & $2.154 \mathrm{Kg}$ & $21.14 \mathrm{~N}$ & $11.622 \mathrm{MPa}$ \\
\hline Carga 3 & $3.154 \mathrm{Kg}$ & $30.95 \mathrm{~N}$ & $19.025 \mathrm{MPa}$ \\
\hline Carga 4 & $4.154 \mathrm{Kg}$ & $40.76 \mathrm{~N}$ & $26.427 \mathrm{MPa}$ \\
\hline
\end{tabular}

De acuerdo con los resultados obtenidos tanto teórica como experimentalmente se observa una aproximación entre los valores arrojados, validando la adquisición de datos por medio de una configuración de roseta en delta.

Tabla 4. Comparación de resultados teóricos y resultados experimentales medidos por el sistema de adquisición de datos

\begin{tabular}{|c|c|c|c|}
\hline & Fuerza & $\begin{array}{c}\text { Esfuerzos Medidos } \\
\text { PXI }\end{array}$ & $\begin{array}{c}\text { Esfuerzos en } \\
\text { ANSYS }\end{array}$ \\
\hline Carga 1 & $11.33 \mathrm{~N}$ & $4.71 \mathrm{MPa}$ & $4.22 \mathrm{MPa}$ \\
\hline Carga 2 & $21.14 \mathrm{~N}$ & $11.24 \mathrm{MPa}$ & $11.622 \mathrm{MPa}$ \\
\hline Carga 3 & $30.95 \mathrm{~N}$ & $18.48 \mathrm{MPa}$ & $19.025 \mathrm{MPa}$ \\
\hline Carga 4 & $40.76 \mathrm{~N}$ & $25.54 \mathrm{MPa}$ & $26.427 \mathrm{MPa}$ \\
\hline
\end{tabular}

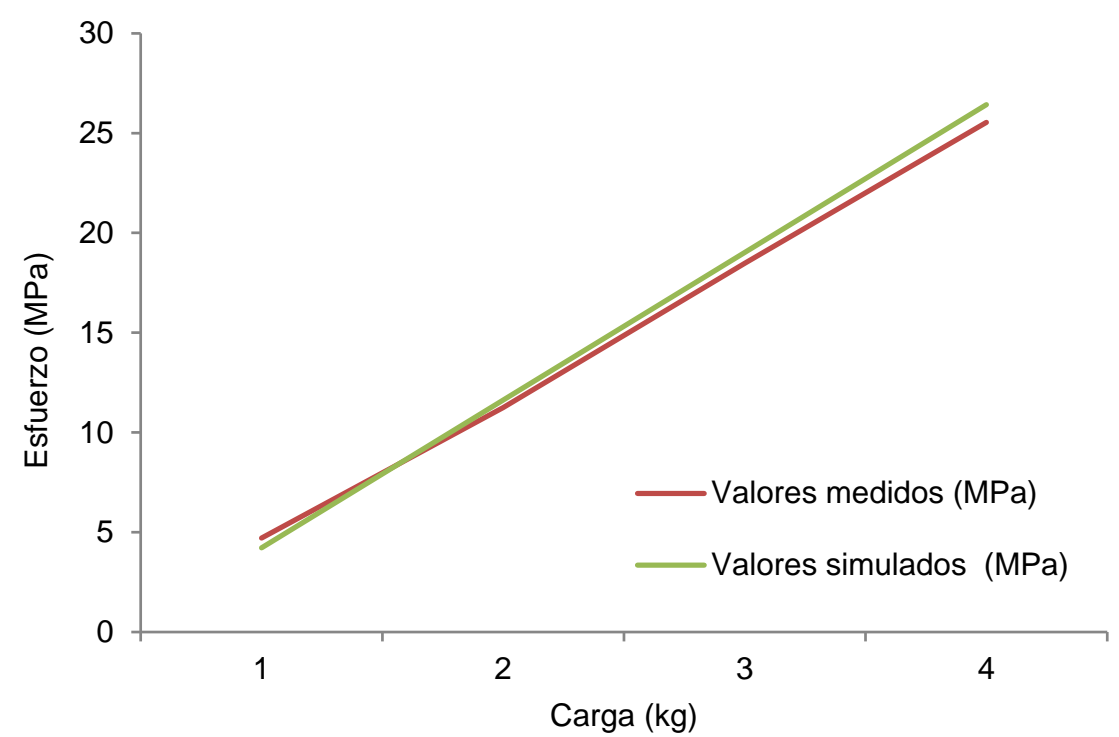

Fig. 7 Comparación de resultados medidos y simulados

En la figura 7 se muestra el comportamiento de los resultados de manera gráfica, donde se puede observar el acercamiento entre los valores simulados y medidos, donde el eje vertical representa el esfuerzo en mega pascales y el eje horizontal las cargas en kilogramos.

\section{CONCLUSIONES}

Por medio de este análisis experimental se comprobó que los equipos de adquisición de datos son de gran utilidad si se adecuan de manera correcta a las necesidades del caso de estudio, pudiéndonos ahorrar tiempo evitando la parte del desarrollo del análisis teórico, en este caso se comprobó mediante tres metodologías diferentes que el porcentaje de error entre ellas es aceptable, por lo que se validó el arreglo de las galgas uniaxiales en configuración roseta delta integradas al sistema de adquisición de datos, proporcionando lecturas confiables con un porcentaje de error promedio del $5 \%$, para el análisis estructural de un perfil aerodinámico NACA 4412. 


\section{REFERENCIAS}

Akça K., Çehreli C. y Iplikçioğlu H., A Comparison of Three Dimensional Finite Element Stress Analysis with In Vitro Strain Gauge Measurements on Dental Implants, Quintessence Pubilshing Co. (2002), http://www.quintpub.com/userhome/ijp/ijp_15_2_Akca1.pdf?origin=publication_detail. Acceso: 10 de Julio (2012).

Escribano M., El Análisis por Elementos Finitos: Una Metodología muy Reciente en Economía, Departamento de Economía Financiera y Contabilidad Escuela Universitaria de Estudios Empresarial Universidad Complutense de Madrid (2007).

Chagoyén C.A., González D., Aragón G.B., Leiva J., La vinculación de la simulación numérica y los ensayos, su aplicación a un puente de ferrocarril, Rev. Tec. Ing. Univ. Zulia, (2013). http://revistas.luz.edu.ve/index.php/rtz/article/view/16384/15804. Acceso: 1 de Marzo (2014)

Gröning, F., Bright, J.A., Fagan, M.J., O'Higgins, P., Improving the Validation of Finite Element Models with Quantitive Full-Field Strain Comparisons, Journal of Biomechanics (2012),

http://www.academia.edu/1454212/Improving_the_validation_of_finite_element_models_with_quantitative_fu II-field_strain_comparisons. Acceso: 3 de Agosto (2013)

Gualda N.J., Sanzi H.C, Ortiz L., Análisis de Tensiones de elementos Finitos de una Válvula Esférica durante la Prueba Hidrostática, y su Verificación Experimental, Rev. Esc. Minas ISSN 0718-0764 2010. http://www.scielo.cl/scielo.php?script=sci_arttext\&pid=S0718-07642010000500006\&lng=es\&nrm=iso.

Acceso: 30 de Agosto (2012)

Kacmarcik J., Vukojevic N. y Hadzikadunic F., Comparison of Numerically and Experimentally Determined SCF for Nozzle in Cylindrical Pressure Vessel, 14th Internacional Research/Expert Conference, (2010), http://www.tmt.unze.ba/zbornik/TMT2010/143-TMT10-053.pdf. Acceso: 13 de Octubre (2012)

King F., El aluminio y sus aleaciones, Limusa Noriga Editores, (1992).

Mandado E., Mariño P., A., Lago. Instrumentación Electrónica. Editorial Alfaomega-Marcombo. (1996).

Manuel A., Biel D., Olivé J., Prat J., Sánchez F.J., Instrumentación Virtual. Adquisición, procesado y análisis de señales. Editorial Alfaomega S. A. de C. V. México D. F. (2002).

Reymer, P., Validation of the FEM Model of the Mi-24 Tail Boom and Vertical Stabilizer, Journal of KONES Powertrain and Transport, (2011), http://ilot.edu.pl/kones/2011/2_2011/2011_reymer_validation.pdf. Acceso: 30 de Septiembre (2012)

Shigley J.E., Mitchell Larry D. Mechanical Engineering Design. Mc Graw Hill. Cuarta edición. México, (1986).

Subauste P R. C., El Desarrollo de las Estructuras Aeronáuticas. (2009).

http://www.scribd.com/doc/15610178/El-desarrollo-de-las-estructuras-aeronauticas. Acceso: 15 Julio (2012)

Watanave J. R. y Urriolagoitia G. C., Curso El Diseño de Maquinaria. 1er Congreso Internacional de la sección de Estudios de Posgrado e Investigación de ESIME, (1996). 